\title{
Nonrigid Registration of 3D Scalar, Vector and Tensor Medical Data ${ }^{\star}$
}

\author{
J. Ruiz-Alzola ${ }^{1,2}$, C.-F. Westin ${ }^{2}$, S.K. Warfield ${ }^{2}$, A. Nabavi ${ }^{2,3}$, and R. Kikinis ${ }^{2}$ \\ 1 Dep. Señales y Comunicaciones. University of Las Palmas de Gran Canaria, Spain \\ 2 Dep. Radiology. Harvard Medical School and Brigham \& Women's Hospital, USA \\ 3 Dep. Neurosurgery. University of Kiel, Germany \\ \{jruiz, westin, warfield, arya, kikinis\}@bwh.harvard.edu
}

\begin{abstract}
New medical imaging modalities offering multi-valued data, such as phase contrast MRA and diffusion tensor MRI, require general representations for the development of automatized algorithms. In this paper we propose a unified framework for the registration of medical volumetric multi-valued data. The paper extends the usual concept of similarity in intensity (scalar) data to vector and tensor cases. A discussion on appropriate template selection and on the limitations of the template matching approach to incorporate the vector and tensor reorientation is also offered. Our approach to registration is based on a multiresolution scheme based on local matching of areas with a high degree of local structure and subsequent interpolation. Consequently we provide an algorithm to assess the amount of structure in generic multi-valued data by means of gradient and correlation computations. The interpolation step is carried out by means of the Kriging estimator that outperforms conventional polynomial methods for the interpolation of sparse vector fields. The feasibility of the approach is illustrated by results on synthetic and clinical data.
\end{abstract}

\section{Introduction}

While there is a large amount of research done on the registration of scalar datasets provided by different types of intensity-based MRI scans and other medical imaging modalities, with a proliferation of algorithms and a solid theoretical background [1], this does not seem to be the case for non-scalar datasets despite their increasing clinical relevance. For example, techniques offering higher dimensional output fields are Phase Contrast Angiography MRI (PCA-MRI), which provides a vector field of blood velocities, or Diffusion Tensor MRI (DT$M R I$ ), which provides a second order symmetric-tensor field description of the

\footnotetext{
* This work has been partially funded by the Spanish Gov. (MEC), visiting research fellowship FPU PRI1999-0175 for the first author, jointly by the European Commission and the Spanish Gov. (CICYT), research grant 1FD97-0881-C02-01, by grant RG 3094A1/T from the National Multiple Sclerosis Society (SKW) and by US grants NIH NCRR P41 RR13218, NIH P01 CA67165 and NIH R01 RR11747. A. Nabavi has been supported by the DFG (NA 365/ 1-1)
} 
local water diffusion in each tissue. The major aim of this paper is to develop a common framework for the three-dimensional registration of scalar, vector and tensor fields that can be readily embedded in medical imaging applications. In particular our goal is to map a reference anatomy, depicted by the signal $S_{r}(\mathbf{x})$, onto a deformed one, represented by the signal $S_{d}(\mathbf{x})$. Both signals are the output of scanning devices and could correspond to different studies of the same patient or to different patients. Equation (1) describes a model to characterize the relationship between both datasets - see also Fig. (1) -, where $D$ models the deformation applied to the reference signal, and both $H$ and the noise $v$ model the inter-scan differences.

$$
S_{d}(\mathbf{x})=H\left[D\left[S_{r}(\mathbf{x})\right]\right]+v(\mathbf{x})
$$

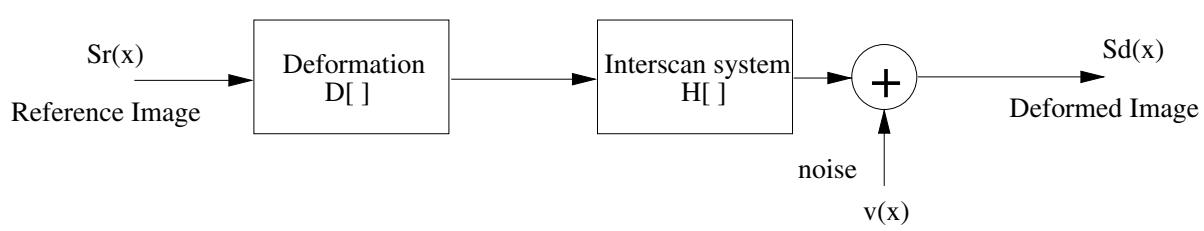

Fig. 1. Model relating two different scans

The deformation $D$ represents a space-variant shift system and, hence, its response to a signal $S(\mathbf{x})$ is $D[S(\mathbf{x})]=S(\mathbf{x}+\mathbf{d}(\mathbf{x}))$. With regard to the inter-scan differences, we are considering $H$ to be a non-memory, possibly space-variant, system depending on a set $\mathbf{h}(\mathbf{x})=\left(h_{1}(\mathbf{x}) \ldots h_{p}(\mathbf{x})\right)^{t}$ of unknown parameters and the noise to be spatially white and with zero mean. With these simplifications and defining $S_{r}^{H}(\mathbf{x})=H\left[S_{r}(\mathbf{x})\right]$, the model (1) reduces to:

$$
S_{d}(\mathbf{x})=S_{r}^{H}(\mathbf{x}+\mathbf{d}(\mathbf{x}))+v(\mathbf{x})
$$

The goal of registration is to find the displacement field $\mathbf{d}(\mathbf{x})$ that makes the best match between $S_{r}(\mathbf{x})$ and $S_{d}(\mathbf{x})$ according to (2). The approach proposed in this paper is based on template matching by locally optimizing a similarity function (Sect. 2). A local structure detector for generic tensor fields (Sect. 3) allows to constrain the matching to areas highly structured. In order to obtain the deformation field in the remaining areas we propose (Sect. 4) an interpolation scheme whose key feature is the probabilistic weighting of the samples using a Kriging Estimator [4] as an alternative to global polynomial models. The whole approach is embedded in a multiresolution scheme using a Gaussian pyramid in order to deal with moderate deformations and decrease the influence of false optima. We also present (Sect. 5) some illustrative results carried out on synthetic and clinical data. 


\section{Template Matching}

Several schemes can be used to estimate the displacement field in (2). When there is no a priori probabilistic information about the signal and noise characterization, a Least-Squares [3] approach is a natural choice. For this all that is required is a suitable definition of an inner product and, thereafter, an induced norm. Note that scalar, vector and tensor fields are applications of a real domain onto Euclidean vector spaces and which allows us to define the inner product between fields by means of the integral over the whole domain of the inner products between their values. Let us consider the functional set $\mathcal{F}=\{f: D \longrightarrow V\}$ where $D$ is a real domain and $V$ is an Euclidean space. Then an inner product can be defined on $\mathcal{F}$ as $<f_{1}, f_{2}>=\int_{D} w(\mathbf{x})<f_{1}(\mathbf{x}), f_{2}(\mathbf{x})>d \mathbf{x}$, where $w(\mathbf{x})$ is a weighting function for the inner product. Note that the inner product in the left-hand side is defined between fields and in the right-hand side, inside the integral, is defined between values of the field.

The least squares estimator is obtained minimizing a cost function (3) that consists of the squared norm of the estimation error.

$$
C^{*}(\mathbf{d}(\mathbf{x}) ; \mathbf{h}(\mathbf{x}))=\left\|S_{d}(\mathbf{x})-S_{r}^{H}(\mathbf{x}+\mathbf{d}(\mathbf{x}))\right\|^{2}
$$

The dependency on the unknown parameters can be removed by estimating them using some constraints. For example, if the parameters are assumed to be constant, a least-squares estimation can be obtained, $\hat{\mathbf{h}}(\mathbf{d})=\hat{\mathbf{h}}\left(S_{d}(\mathbf{x}), S_{r}(\mathbf{x}+\right.$ $\mathbf{d}(\mathbf{x}))$ ), and substituted in $C^{*}$ to obtain a new cost function (4) that only depends on $\mathbf{d}(\mathbf{x})$.

$$
C(\mathbf{d}(\mathbf{x}))=C^{*}(\mathbf{d}(\mathbf{x}) ; \hat{\mathbf{h}}(\mathbf{x}))
$$

The optimization of $C(\mathbf{d}(\mathbf{x}))$ in order to obtain the displacement field $\mathbf{d}(\mathbf{x})$ is a daunting task that requires additional constraints to make it feasible. Template Matching trades off accuracy and computational burden to approximate a solution for this optimization problem. In particular it assumes that (2) holds with a uniform displacement field (i.e. translational) and constant parameters in a neighborhood of the point $\mathbf{x}_{0}$ to be corresponded. This prevents the template matching method to be successfully used in the presence of significant rotational deformation fields. There is a fundamental trade-off to be considered in the design of the neighborhood: it must be non-local, and hence large in size, in terms of the $S_{d}(\mathbf{x})$ space-frequencies to avoid the ill-posedness arising from the lack of discriminant structure (aperture problem [2]), and it must be local, and hence small in size, in terms of the unknown displacement field spatial-frequencies to guarantee invariance of the transformation parameters. If the template is too small, the problem remains ill-posed and the contribution of noise leads to false solutions. Conversely, if the template is too large the estimated parameters for the transformation are a smoothed version of the actual ones. Adaptive templates with different sizes and weights can help to deal with this problem.

Let $T\left(\mathbf{x}-\mathbf{x}_{0}\right)$ be a window function centered in a generic point $\mathbf{x}_{0}$ in the deformed dataset and designed following the previous remarks. The template 
matching assumptions transform (2) into (5), that holds for every point $\mathbf{x}_{0}$ in the deformed dataset.

$$
T\left(\mathbf{x}-\mathbf{x}_{0}\right) S_{d}(\mathbf{x})=T\left(\mathbf{x}-\mathbf{x}_{0}\right) S_{r}^{H}\left(\mathbf{x}+\mathbf{d}\left(\mathbf{x}_{0}\right)\right)+v(\mathbf{x})
$$

Equation (5) has an intuitive interpretation: for every point $\mathbf{x}_{0}$ in the deformed dataset a template $T\left(\mathbf{x}-\mathbf{x}_{0}\right) S_{d}(\mathbf{x})$ is constructed and compared against every possible template around it in the transformed (by $H$ ) reference dataset. This means that a complex global optimization problem has been split into many simple local ones. In order to constrain the variability of the estimated displacement field it can be smoothed after the matching using a linear filter.

\subsection{Similarity Functions}

A Similarity Function is any convenient monotonic function of the cost (3), $S(\mathbf{d}(\mathbf{x}))=F[C(\mathbf{d}(\mathbf{x}))]$, that leaves the locations of the optima unchanged. Under the template matching assumptions the similarity function $S\left(\mathbf{d} ; \mathbf{x}_{0}\right)$ is local and, hence, it depends on $\mathbf{x}_{0}$. Here the least-squares method referred to above is used to obtain suitable local similarity functions for the template matching of generic tensor fields.

Let us first consider that $H$ is the identity mapping and, hence, (3) and (4) are equal. Direct use of (4) provides the similarity function (6) that corresponds to the well-known Sum of Squared Differences.

$$
S_{S S D}\left(\mathbf{d} ; \mathbf{x}_{0}\right)=\left\|T\left(\mathbf{x}-\mathbf{x}_{0}\right)\left(S_{d}(\mathbf{x})-S_{r}(\mathbf{x}+\mathbf{d})\right)\right\|^{2}
$$

The extension of (6) using inner products and assuming that $\left\|S_{r}(\mathbf{x}+\mathbf{d})\right\|^{2}$ is almost constant for all possible $\mathbf{d}$ leads to an alternative similarity that corresponds to the Correlation measure.

$$
S_{C}\left(\mathbf{d} ; \mathbf{x}_{0}\right)=<S_{d}(\mathbf{x}), S_{r}(\mathbf{x}+\mathbf{d})>
$$

Let us now consider that $H$ is an affine transformation of the intensity. In this case $S_{r}^{H}(\mathbf{x}+\mathbf{d})=a S_{r}^{H}(\mathbf{x}+\mathbf{d})+b$ and the cost (3) turns out to be (8)

$$
C^{*}\left(\mathbf{d} ; a, b ; \mathbf{x}_{0}\right)=\left\|T\left(\mathbf{x}-\mathbf{x}_{0}\right)\left(S_{d}(\mathbf{x})-a S_{r}(\mathbf{x}+\mathbf{d})-b\right)\right\|^{2}
$$

Minimizing (8), expanding the inner product, it is not difficult to find an estimator for the parameters $a$ and $b$. Substituting them into (8) and making some manipulations its possible to derive the well-known correlation coefficient similarity measure (9), whose absolute value is to be maximized and where $\mathbf{s}=T\left(\mathbf{x}-\mathbf{x}_{0}\right) S_{d}(\mathbf{x}), \mathbf{p}_{1}=T\left(\mathbf{x}-\mathbf{x}_{0}\right) S_{r}(\mathbf{x}+\mathbf{d}), \mathbf{p}_{2}=T\left(\mathbf{x}-\mathbf{x}_{0}\right)$ and $\hat{\mathbf{p}}_{\mathbf{2}}=\frac{\mathbf{p}_{2}}{\left\|\mathbf{p}_{2}\right\|}$. The correlation coefficient provides a geometric interpretation as the cosine of the angle between the normalized versions of $T\left(\mathbf{x}-\mathbf{x}_{0}\right) S_{d}(\mathbf{x})$ and $T\left(\mathbf{x}-\mathbf{x}_{0}\right) S_{r}(\mathbf{x}+\mathbf{d})$, obtained removing the mean value and making the signals to have unit norm.

$$
S_{\rho}\left(\mathbf{d} ; \mathbf{x}_{0}\right)=\left\langle\frac{\mathbf{s}-<\mathbf{s}, \hat{\mathbf{p}}_{2}>}{\left\|\mathbf{s}-<\mathbf{s}, \hat{\mathbf{p}}_{2}>\right\|}, \frac{\mathbf{p}_{1}-<\mathbf{p}_{1}, \hat{\mathbf{p}}_{2}>}{\left\|\mathbf{p}_{1}-<\mathbf{p}_{1}, \hat{\mathbf{p}}_{2}>\right\|}\right\rangle
$$


The application of the equations above requires a proper definition of the inner product (10) and its induced norm (11). We assume that the tensors are cartesian (defined with respect to an orthonormal basis) and we are using the Einstein notation for sums (any repetition of an index entails a summing over this index). Note that with these definitions the elements with the form $\left.\mathbf{f}-<\mathbf{f}, \hat{\mathbf{p}}_{2}\right\rangle$ in (9) are the mean value of $\mathbf{f}$ weighted by $T\left(\mathbf{x}-\mathbf{x}_{0}\right)$.

$$
\begin{aligned}
<S_{1}(\cdot), S_{2}(\cdot) ; \mathbf{x}_{\mathbf{0}}> & =\int_{D} S_{1_{i_{1} \ldots i_{n}}}(\mathbf{x}) S_{2_{i_{1} \ldots i_{n}}}(\mathbf{x}) d \mathbf{x} \\
\left\|S(\cdot) ; \mathbf{x}_{\mathbf{0}}\right\|^{2} & =\int_{D} S_{i_{1} \ldots i_{n}}(\mathbf{x}) S_{i_{1} \ldots i_{n}}(\mathbf{x}) d \mathbf{x}
\end{aligned}
$$

\subsection{Warped Vectors and Tensors}

Vector and tensor data are linked to the body under inspection and, thereafter, any warping of the supporting tissue will lead to a consequent warping or reorientation of these data. In fact, as far as the function and the domain share the same reference system (or any rigidly related one), any transformation applied to the domain will affect to the function too. In particular, we are considering the domain transformation to be analytic and, hence, to preserve local topology, i.e., differential line elements map onto differential line elements, the same being true for differential surface and volume elements; moreover, differential ellipsoids are mapped onto differential ellipsoids. The warping of the domain can be expressed by the transformation $\mathbf{x}=\mathbf{T}\left(\mathbf{x}^{\prime}\right)$, where $\mathbf{x}$ stands for points in the reference dataset and $\mathbf{x}^{\prime}$ for points in the deformed one. The differential of the transformation is

$$
d \mathbf{x}=\left[\nabla \otimes \mathbf{T}\left(\mathbf{x}^{\prime}\right)\right] d \mathbf{x}^{\prime}
$$

where the tensor product $\otimes$ has been used to define the deformation gradient $\mathbf{A}\left(\mathbf{x}^{\prime}\right)=\left[\nabla \otimes \mathbf{T}\left(\mathbf{x}^{\prime}\right)\right]$, which can be recognized as the Jacobian matrix of the mapping. Consequently, two vectors $\mathbf{v}$ and $\mathbf{v}^{\prime}$ and two second order tensors $\mathbf{P}$ and $\mathbf{P}^{\prime}$ are locally related as follows:

$$
\begin{aligned}
\mathbf{v} & =\left[\nabla \otimes \mathbf{T}\left(\mathbf{x}^{\prime}\right)\right] \mathbf{v}^{\prime} \\
\mathbf{P} & =\left[\nabla \otimes \mathbf{T}\left(\mathbf{x}^{\prime}\right)\right]^{-1} \mathbf{P}^{\prime}\left[\nabla \otimes \mathbf{T}\left(\mathbf{x}^{\prime}\right)\right]
\end{aligned}
$$

The Polar Decomposition Theorem [5] states that for any non-singular square matrix, such as the Deformation Gradient $\mathbf{A}\left(\mathbf{x}^{\prime}\right)$, there are unique symmetric positive definite matrixes $\mathbf{U}\left(\mathbf{x}^{\prime}\right)$ and $\mathbf{V}\left(\mathbf{x}^{\prime}\right)$ and also an unique orthonormal matrix $\mathbf{R}\left(\mathrm{x}^{\prime}\right)$ such that $\mathbf{A}\left(\mathrm{x}^{\prime}\right)=\mathbf{R}\left(\mathrm{x}^{\prime}\right) \mathbf{U}\left(\mathrm{x}^{\prime}\right)=\mathbf{V}\left(\mathrm{x}^{\prime}\right) \mathbf{R}\left(\mathrm{x}^{\prime}\right)$. This leads to important geometric interpretations of the geometric mapping. For example, notice that a sphere is first stretched by the mapping in the directions of the eigenvectors of $\mathbf{U}\left(\mathbf{x}^{\prime}\right)$ and then rotated by $\mathbf{R}$. Thereafter, a transformation such that locally $\mathbf{R}\left(\mathbf{x}^{\prime}\right)=\mathbf{I}$ is said to be a Pure Strain at $\mathbf{x}^{\prime}$ while if $\mathbf{U}\left(\mathbf{x}^{\prime}\right)=\mathbf{V}\left(\mathbf{x}^{\prime}\right)=\mathbf{I}$ it is said to be a Rigid Rotation at that point. 
It was said above (Sect. 2) that the matching approach to data registration relies on the fact that the displacement field is constant inside the matching template. This imposes a hard local constraint on the deformation gradient, since it must locally be close to the identity matrix. Therefore, unless the template is reduced to a single point (for example, by smoothing and downsampling) one should not include the local directional change of the vector and tensor fields due to the domain warping in the matching problem. Nevertheless, once the displacement field has been estimated, it is possible to use (13) and (14) to compute the reorientation of the vector or tensor field.

\section{Structure Measures}

Matching must be constrained to areas with local high discriminant structure in order to be successfully applied, making sure that the local similarity function are narrow around the optima. We propose to threshold a convenient measure of cornerness to identify the appropriate areas. In the scalar case some of these measures are based on identifying points corresponding to curved edges by means of the locally averaged outer product (i.e. the correlation matrix) of the gradient field [6]. The gradient of a tensor field is expressed in invariant form with a tensor product $\nabla \otimes S(\mathbf{x})$. Using an orthonormal basis, the gradient of a scalar field in any point is a vector whose components are the partial derivatives of the function. For a vector field, the gradient turns out to be the Jacobian matrix with the partial derivatives for each component and for a second order tensor field it is a $3 D$ array with the partial derivatives of every component of the tensor. The comma [5] convention becomes very handy to represent gradients in component form and it has been used in (15), where, $k$ indicates an indexing of the partial derivatives and $n$ is the order of $S$. Note that for each component of a generic field we obtain a gradient vector that is arranged into the overall gradient tensor.

$$
\nabla \otimes S(\mathbf{x})=S_{i_{1} \ldots i_{n}, k}(\mathbf{x})
$$

In the scalar case, the correlation matrix of the gradient vector, i.e., the symmetric, positive semidefinite second order tensor formed by the mathematical expectation of the outer product of the gradient vector (16) provides the basis to assess cornerness analyzing its associated quadratic form (ellipsoid): The rounder the ellipsoid the bigger the cornerness while a very elongated ellipsoid would indicate a straight edge.

$$
\mathbf{R}_{\nabla \mathbf{S}}(\mathbf{x})=E\{\nabla S(\mathbf{x}) \otimes \nabla S(\mathbf{x})\}
$$

In order to extend this idea to the vector and tensor cases, note first that (16) can be directly extended into the correlation tensor (17).

$$
\mathbf{R}_{\nabla \mathbf{S}}(\mathbf{x})=E\{\nabla \otimes S(\mathbf{x}) \otimes \nabla \otimes S(\mathbf{x})\}
$$

For example, if we had a second order tensor field we would obtain a sixth order correlation tensor. The generalized correlation matrix (18) is defined as 
the contraction of all the non-differential components in (17) with respect to an orthonormal basis.

$$
\mathbf{R}_{\nabla \mathbf{S}}(\mathbf{x})=E\left\{S_{i_{1} \ldots i_{n}, k}(\mathbf{x}) S_{i_{1} \ldots i_{n}, l}(\mathbf{x})\right\}
$$

Due to the linear nature of the expectation operator, the generalized correlation matrix of the gradient of a generic tensor field is the sum of the correlation matrices of the gradient vectors of each coordinate. This is consistent with the fact that each component adds its own edge and, therefore, the ellipsoid associated to the generalized correlation matrix get rounder as new components are added unless they do not provide additional directional information.

Matching is therefore constrained to points where the ellipsoids associated to the generalized correlation matrices are large and round enough, meaning strong and curved edge.

\section{Interpolation: The Kriging Estimator}

The structure analysis carried out in the previous section provides a sparse set of clusters of $3 D$ points on which a local estimation of the displacement can be performed by template matching. Two comments must be made in order to interpolate the displacement field in the remaining areas. First of all the field can be discontinuous due to the relative motion between different bodies; this prevents the direct use of any global polynomial interpolation approach since no single model can fit everywhere in the dataset. Second, the displacement field components on the boundaries of touching organs must have continuous components in the directions normal to the surface interfaces, though the field can be discontinuous in the orthogonal tangent plane. This simply indicates that the motion at the boundaries can be decomposed in a discontinuous sliding component and in a continuous pushing one.

The Kriging Estimator (KE), which originated in geostatistics [7,8], is a method to deal with spatially dependent data. Essentially it provides a linear estimator of an unknown sample of a random field using a set of known samples. The estimator is designed to minimize the mean squared error under the constraint of the the estimator to be unbiased, i.e., the weights must sum up one. From a practical point of view, the $K E$ weighs the contribution of each known sample according to its distance to the one to be estimated. This means that distant samples lose importance and eventually can be ignored in the estimator while closer samples, which are more likely to be part of the same body and to have similar realization values, increase their relative importance. Its application to the interpolation of $3 D$ scalar medical images has been referred elsewhere [9]. Here the method is used in the interpolation of displacement fields considering each spatial component independently. A hypothesis is made about a so-called variogram function, which is the mean squared error between two samples at a distance $r$. See [4] for a discussion on different variograms. Essentially a parametric model is proposed as theoretical variogram and parameter 
estimation from the known data using, for example, least-squares, leads to the experimental variogram, which is the one actually used.

The $K E$ interpolator provides an estimator for the components $d_{i}\left(\mathbf{x}_{0}\right)$ of the displacement field in $\mathbf{x}_{0}$ as a linear combination of the corresponding components matched at the high structure locations $d_{i}\left(\mathbf{x}_{j}\right)$, as it is indicated in (19).

$$
d_{i}\left(\mathbf{x}_{0}\right)=\sum_{j=1}^{N} h_{j} d_{i}\left(\mathbf{x}_{j}\right)
$$

The weights of the estimator are obtained solving the linear system of equation (20), where $\gamma\left(\left\|\mathbf{x}_{i}-\mathbf{x}_{j}\right\|\right)$ is the evaluation of the variogram between point $\mathbf{x}_{i}$ and $\mathbf{x}_{j}$.

$$
\left(\begin{array}{ccccc}
0 & \gamma\left(\left\|\mathbf{x}_{2}-\mathbf{x}_{1}\right\|\right) & \ldots \gamma\left(\left\|\mathbf{x}_{N}-\mathbf{x}_{1}\right\|\right) & 1 \\
\gamma\left(\left\|\mathbf{x}_{2}-\mathbf{x}_{1}\right\|\right) & 0 & \ldots & \gamma\left(\left\|\mathbf{x}_{N}-\mathbf{x}_{2}\right\|\right) & 1 \\
\vdots & \vdots & \vdots & \vdots & \vdots \\
\gamma\left(\left\|\mathbf{x}_{N}-\mathbf{x}_{1}\right\|\right) & \gamma\left(\left\|\mathbf{x}_{N}-\mathbf{x}_{2}\right\|\right) & \ldots & 1 & 0 \\
1 & 1 & \ldots & 1 & 0
\end{array}\right)\left(\begin{array}{c}
h_{1} \\
h_{2} \\
\vdots \\
h_{N} \\
\mu
\end{array}\right)=\left(\begin{array}{c}
\gamma\left(\left\|\mathbf{x}_{0}-\mathbf{x}_{1}\right\|\right) \\
\gamma\left(\left\|\mathbf{x}_{0}-\mathbf{x}_{2}\right\|\right) \\
\vdots \\
\gamma\left(\left\|\mathbf{x}_{0}-\mathbf{x}_{N}\right\|\right) \\
1
\end{array}\right)
$$

\section{Results}

The framework presented in this paper is under evaluation in a number of clinical cases at Brigham \& Women's Hospital. The implementation relies on sampled data and therefore the integrals in (10) and (11) become sums. The window function $T(\mathbf{x})$ is defined to be the unity inside a cube centered in the origin and zero outside. The local structure detection is based on (18) where the expectation is computed from the sample mean. Since $\operatorname{det}\left(\mathbf{R}_{\nabla S}(\mathbf{x})\right)=\prod \lambda_{i}$ and $\operatorname{tr}\left(\mathbf{R}_{\nabla S}(\mathbf{x})\right)=\sum \lambda_{i}\left(\lambda_{i}\right.$ are the eigenvalues of $\left.\mathbf{R}_{\nabla S}\right)$, only points above a threshold in $t_{1}(\mathbf{x})=\frac{\operatorname{det}\left(\mathbf{R}_{\nabla S}(\mathbf{x})\right)}{\operatorname{tr}\left(\mathbf{R}_{\nabla S}(\mathbf{x})\right)}$ are considered. False detections due to high gradients are avoided thresholding $t_{2}(\mathbf{x})=\frac{\operatorname{det}\left(\mathbf{R}_{\nabla S}(\mathbf{x})\right)}{\operatorname{tr}\left(\frac{1}{N} \mathbf{R}_{\nabla S}(\mathbf{x})\right)^{N}}$ that varies between zero and one depending on the shape of the associated ellipsoid, with one meaning a perfect sphere. The structure detector is only applied to the deformed dataset, providing clusters of points in which the displacement vector is to be estimated using template matching around a neighborhood. The warp is interpolated in a generic point of low structure using Kriging derived from the N closest highly structured points with known displacement. The estimated warp is finally smoothed with a linear filter. The full approach is embedded in a gaussian pyramid so that warps estimated at coarser resolution levels are linearly interpolated in order to be used as initial displacement in the next higher resolution level.

Figure (2.a) shows a $32 \times 32 \mathrm{MRI}$ slice of the corpus callosum that is deformed by a synthetic gaussian field as depicted in Fig. (2.b). In Fig. (2.c) it is shown the correctly estimated displacement field in areas of high structure (blue arrows) and the interpolated field (red) using the Kriging estimator with a linear 
variogram $\gamma(r)=a r$. All experiments carried out have confirmed the superior performance of the Kriging estimator over polynomial interpolators both with synthetic and real deformations.Figure (3.a) shows an axial slice of a DT-MRI dataset ${ }^{1}$ of the corpus callosum where the principal eigenvectors directions have been represented using a color coding ranging from blue (in-plane projection) to red (orthogonal to plane) [10]. The whole approach has been applied to warp this dataset into another corresponding to a different individual, shown in Fig. (3.b), using three levels of a gaussian pyramid, local templates of dimension $3 \times 3$ and a linear variogram for the Kriging interpolator that is limited to take into account the 20 closest samples. Figure (3.c) shows a T2W zoomed version of the righthand side of the former, corresponding to the posterior corpus callosum and the estimated deformation field.

\section{Conclusions}

We have presented a unified framework for non-rigid registration of scalar, vector and tensor medical data. The approach is local, since it is based on templatematching, and resorts to a multirresolution implementation using a Gaussian pyramid in order to provide a coarse-to-fine approximation to the solution which allows to deal with moderate deformations and avoids false local solutions. The method does not assume any global a priori regularization and, therefore, avoids the computational burden associated to those approaches. We also have extended the concept of discriminant structure to the tensor case, providing a new operator to detect it. The Kriging Estimator outperforms polynomial approaches for the interpolation of sparse displacement fields and to the best of our knowledge this is the first time to be used with this purpose in medical image analysis. The whole approach is under evaluation in a number of clinical cases at Brigham \& Women's Hospital with preliminary promising results.

\section{References}

1. A. Roche et al. Towards a Better Comprehension of Similarity Measures Used in Medical Image Registration. Proc. MICCAI'99, pp. 555-566, Cambridge, UK, 1999

2. T. Poggio, V. Torre, and C. Koch. Computational vision and regularization theory. Nature, vol. 317, pp. 314-319, sept. 1985

3. T. K. Moon and W. W. Stirling. Matehematical Methods and Algorithms for Signal Processing, Prentice-Hall, NJ, 2000

4. J.L. Starck, F. Mrtagh and A. Bijaoui. Image Processing and Data Analysis. The Multiscale Approach, Cambridge University Press, UK, 1998

\footnotetext{
${ }^{1}$ GE Signa 1.5 Tesla Horizon Echospeed 5.6 scanner, Line Scan Diffusion technique,1 $\mathrm{min} /$ slice, no averaging, effective $\mathrm{TR}=2.4 \mathrm{~s}, \mathrm{TE}=65 \mathrm{~ms}, b_{\text {high }}=750 \mathrm{~s} / \mathrm{mm}^{2}$, fov $=22$ $\mathrm{cm}$, voxel size $4.8 \times 1.6 \times 1.5 \mathrm{~mm}^{3}, 6 \mathrm{kHz}$ readout bandwidth, acquisition matrix $128 \times 128$
} 


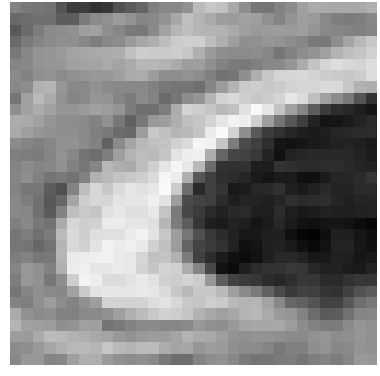

a)

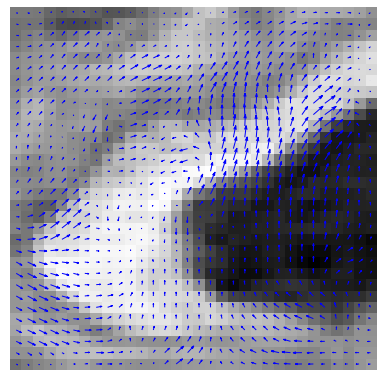

b)

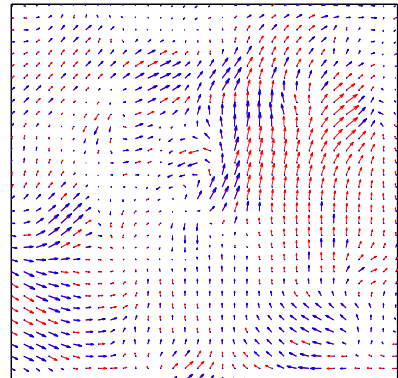

c)

Fig. 2. Displacements fields (b) real (c) blue: estimated, red: Kriging interpolated

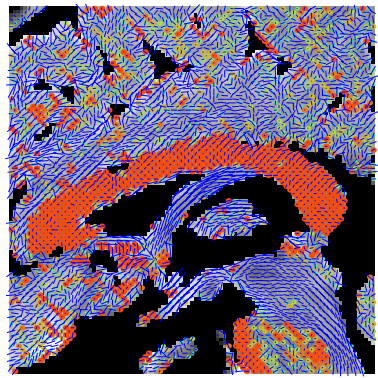

a)

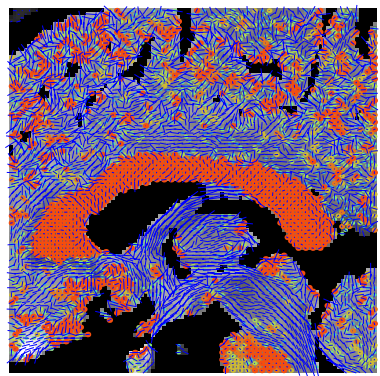

b)

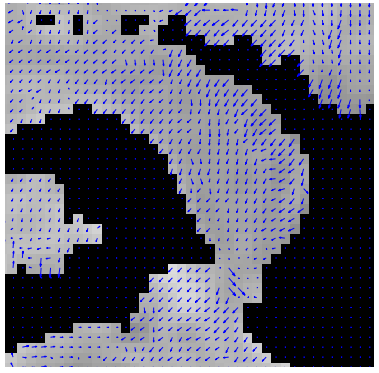

c)

Fig. 3. DT-MRI interpatient warping. a, b) Axial DTMRI of different individuals. c) zoomed $\mathrm{T} 2 \mathrm{~W}$ of the posterior corpus of a) and estimated deformation

5. L.A. Segel. Mathematics Applied to Continuum Mechanics, Dover, NY, 1987

6. K. Rohr. On 3D Differential Operators for Detecting Point Landmarks. Image and Vision Computing, 15:219-233, 1997

7. D. Krige. A Statistical Approach to Some Mine Valuation and Allied Problems on the Witwatersrand, Master Thesis, University of Witwatersrand, 1951

8. N. Cressie. Kriging Nonstationary Data. Journal of the American Statistical Association, 81:625-634, 1986

9. R. W. Parrott et al. Towards Statiscally Optimal Interpolation for 3D Medical Imaging. IEEE Engineering in Medicine and Biology, pp. 49-59, sept. 1993

10. S. Peled et al. Magnetic Resonance Imaging Shows Orientation and Asymmetry of White Matter Tracts. Brain Research, vol. 780, pp. 27-33, jan. 1998 